\title{
Hybrid Model to Reduce Latency in Vertical Hand Off
}

\author{
Muhammad Faisal \\ Department of Computing, \\ Shaheed Zulfikar Ali Bhutto Institute of Science \& \\ Technology
}

\author{
Muhammad Ibrahim \\ Department of Computing \\ Virtual university (VU), Islamabad, Pakistan \\ (SZABIST), Islamabad, Pakistan.
}

\begin{abstract}
As the number of network service provider's increases so the numbers of handoffs are increases to avail the best quality of services within affordable cost. In such scenarios fast and secure handoff is obligatory but diversification always creates worries of intruders and latency due to heterogeneous architectures with multiple access points. And elongated latency may go ahead to jitter, ping pong effect, triangular routing and packet loss like issues. Neither the reactive nor the proactive protocols alone for vertical hand off addresses the problem of latency efficiently due to their onboard calculation during peak time of handoff. Ensuring security in such frequent vertical handover without interrupted and delayed services is challengeable but desirable as well. Here we proposed a hybrid model for vertical handoff dividing the standard IEEE 802.11 hand off phases in reactive and proactive manner. Initially executing triggering, discovery and authentication phases in proactive mode while association, IP acquisition and home agent acquisition phases in reactive. That will eventually results in reducing latency and packet loss in the best possible way for vertical handoff while maintaining effective continuous session and provide reliable services. For performance evaluation in our scheme we used reactive (AODV), proactive (OLSR) and hybrid (TORA) protocols via OPNET simulator. Parameters used are throughput, end-to-end delay and packet delivery ratio. The results show that hybrid protocol using less time comparatively with either reactive or proactive protocols.
\end{abstract}

Keywords: Handoff, reactive protocols, proactive protocols, hybrid protocols, latency, jitter, triangular routing, ping pong effect, packet loss

General Terms: House agent registration, IP acquisition, association, authentication, discovery and Triggering.

\section{INTRODUCTION}

The handover between heterogonous ${ }^{1}$ networks provide many challenges [1]. But the most challenging task is the overall handover latency of target networks which ultimately leads to packet loss. The mobile nodes are floating in the same network (homogenous) or in different architecture network (heterogonous) facing various sorts of issues and needs specific requirements for its fulfillment. To handle dynamic connectivity and providing guaranteed services both of these networks need a dynamic and efficient scheme. In situations such as audio, video and real time data transmission these networks needs to provide quality of service (QoS). But this is a challenging task because of every time change in the premises of the network. To avoid packet loss, jitter, ping pong effect reducing latency is mandatory. Mobile node while scanning the surrounding networks for continuity of service having the option of all available networks for handoff but will decide on the RSSI strength value [1].
While analyzing the latency problem in horizontal hand off, the overall time consumed by either reactive or proactive protocols is the summation of the processing of each and every step of IEEE802.11 handover process [2]. Triggering will be activated as the threshold value for the mobile node is concurred as the mobile node is going far away from its access point. As the RSSI value is decreased the mobile node is searching for the new access point of the required RSSI value to persist his continuity of service. After discovering the RSSI value suitable for the mobile node either by active or passive scan the node is now willing for handoff. In active scan the mobile node initiate the scan and request with the access point while in passive scan the mobile node just eavesdropping the signal of the access point. To achieve authentication the mobile node requesting the switching node or access point control center for attachment [2]. The control room authenticates the mobile node either by shared or open system authentication. Now to gain association the mobile node sends an association request to the access point with its old access point association data records. For IP acquisition the mobile node replied with router solicitation message in response to router advertisement message of the access point. The mobile node creates a Care of Address $(\mathrm{CoA})$ which is verified by duplicate address detection (DAD) procedure at the access point control centre. In the last the house agent registration is mandatory for the node to inform its parent house about its CoA to remain in contact with each other through binding update message and binding acknowledgement message respectively [2]. Co-existence of multiple networks make easy to mobile nodes frequent handoff. This speedy and unjustifiable handoff from one network to another originated by erroneous judgments which is called ping pong effect [4]. Resultantly it generates effects like packet loss, useless energy utilization, service disruption, minimizing mobile terminal performance and enhanced the network load. However several efforts have been done to control the effect. Major strategies for its counter measure are probability based handoff and MADM (Multiple attributes Decision Making) [4]

In this paper we will presents in the forthcoming section 2 the whole background of the vertical handover using different scenarios, in section 3 our data flow model is discussed, in section 4 mathematical calculation is verified, in section 5 algorithm is inscribed about each and every phase to be executed in the hybrid model. Section 6 our conceptual model is evaluated with past deficiencies and future enhancements, in section 7 simulation results are presented and discussed. In the last but not the least the in section 7 concluding remarks are given and future working areas are identified.

\section{BACKGROUND STUDY}

Handoff sometimes called two layers handoff or L2 and L3 handoff [13]. Because all its phases are executing in two main layers of $\mathrm{OSI}^{2}$ (open system interconnection) model they network layer (L3) and data link layer [13]. Triggering, 
discovery, authentication and association on data link layer (L2) while IP acquisition and house agent registration are executing on network layer (L3).As shown in the following diagram [1][2][13].

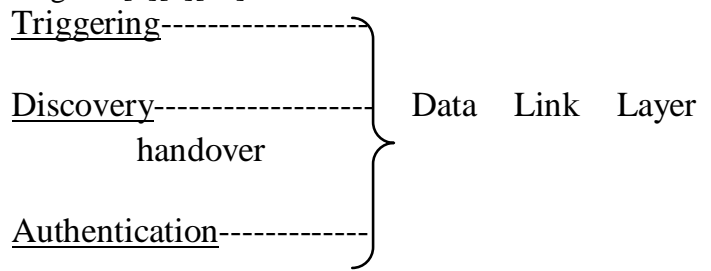

Association-

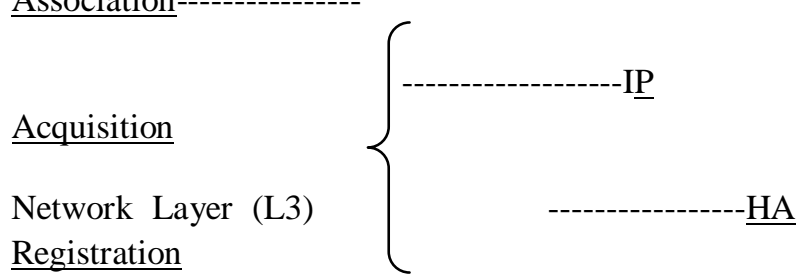

Handover

\section{Figure1.1Architectural diagram}

In handoff user's having the facility to utilize all the available heterogeneous networks, based on cost, throughput, bandwidth, security, user mode and necessity. In both static and dynamic case the mobile node can access the spectrum of all available networks. As shown in the figure.

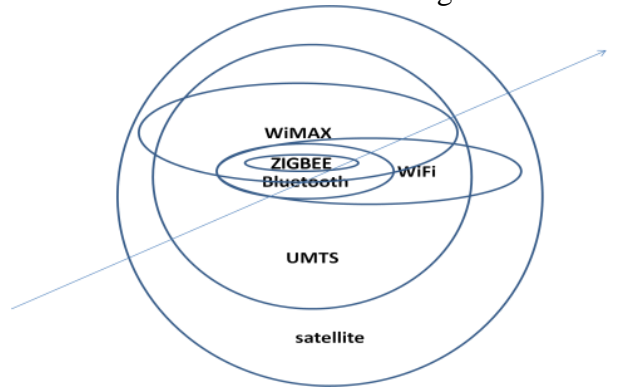

Figure 1.2 User travelling trajectory

\section{LITERATURE REVIEW /RELATED WORK}

In all available scenarios till date the most successful handoff executed are scrutinized and discussed here with the pros and cons of each and every paper studied.

Hoyeon Lee et al [5], put forward improved session initiation protocol paradigm for vertical handoff between UMTS-toWiFi to diminish the IP Multimedia Subsystem (IMS) ${ }^{3}$ delay while comparing the new results with the traditional session initiation protocol [5]. The author's implemented makebefore-break vertical handoff for IMS, introduces two new headers in $\mathrm{SIP}^{4}$, supporting real-time applications which are delay sensitive, showing compatibility with conventional SIP [5]. But the author's ignored the latency created in layer 2 and layer 3 handoff and did not concerned for the emerging difficulties on bandwidth due to enlargement in header size [5].

In [6] the layer2 and layer3 signaling messages merged to contain vertical handoff latency, packet loss ratio in UDP and the TCP throughput enhancement. But here the author's recommended the predictive approach for fast handoff where the probability of success is not always sure [6]. If the predictive approach is replaced with reactive or proactive handoff it will better tackle the latency issue [6].

The authors Neila KRICHENE et al [7] recommended an authentication scheme for vertical handoff among four distinct networks GSM, UMTS, WiFi and WiMAX knocking out any earlier subscription of mobile node for inter or intra networks. The author's proved the resistance of the authentication scheme for denial of service attacks and MIM attack. But this work is only limited for mesh topology in $4 \mathrm{G}$ networks and doesn't take care for quality of service during vertical handoff [7].

The paper [8] described a vertical handoff among WiFi, WiMAX and UMTS networks. Without losing connectivity, using EAP3 and IKE version2 for authentication and dynamic IP allocation respectively, the author's successfully plasticized the authentication in vertical handoff on vehicular networks. But the author's did not discuss the vertical handoff in overlapping no overlapping zone on vehicular network. Both mobility and security strategies are not allocated the appropriate interfaces [8].

Chan-Kyu Han et al [9] reviewed the vertical handoff interoperability in evolved packet system design networks fallowed by its authentication procedures and signaling load capability. The author's permitting processes authentication randomly and make capable the authentication signals strong enough as required for the network. But the author's ignored the necessary scenarios in vertical handoff such as mobility organization neither assigned the handoff in reactive nor proactive manner. Similarly the future of randomly authentication processes in real world implementation and its consequences on security concerns [9].

For efficient vertical handoff in different wireless networks recommended Host Identity Protocol through an incorporated design in paper [10]. The solution can resolve bitterly the multi-homing problem in mobility. Secondly the author also implemented the diameter protocol for user's authentication. But the author used the local scope identifier (LSI) for host identity which is a 32-bit identifier so resultantly the compatibility issue with IPv6 will be raised. And probability of collision will be happened; hence it will be limited in scope [10].

In paper [11] author's tried to combine both vertical handoff and authentication in one module for real time data and compare its results with IP multimedia subsystems (IMS), mobile IPv6 (MIPv6) fast handoff for mobile IPv6 (FMIPv6) respectively. In this way the handoff latency is reduced to nominal way. But the author's did not chalk out any strategy for the packet loss scenario. Any packet loss scenario will jerk the system to resynchronization. Either the data communication will re-start the new handoff association or may be turn reverse to the starting point [11].

The author's Victor C. M. Leung and Ali Al Shidhani [12] to minimize the re-authentication in handoff between UMTSWLAN planned FAUR-LFR instead of already implemented EAP-EKE, UMTS-AKA. The results restricted the handoff delay and signaling traffic to a satisfactory level, without any re-adjustment in the networks architecture. But the FAUR's security keys have lifetime validity, which is the most unsecure strategy in information security management field [12].

While considering handoff in almost every sort of network architecture in the literature review we

observed one common concern in all types of cons in the research papers that is the latency issue. Whenever the latency issue is raised it gives birth to many other issues as well like jitter, ping-pong effect, triangular routing and packet loss. If the latency problem is resolved efficiently then the birth of all 
the rest issues will be minimized instead of the occurrence probability.

\section{Conceptual model}

In our conceptual model we summarized the past deficiencies on the basis of which we proposed our novel conceptual idea for the latency solution. After that providing discussions and proofs for the proposed new conceptual model validating the results with OPNET simulator.

\section{Problem Statement}

According to the IEEE802.11 standard six steps are executed while horizontal Hand Off is underway. They are HA registration, IP acquisition, association, authentication, discovery and Triggering in reverse order [3] [13] [2] [1]. The standard IEEE handoff and all its variants almost based on either proactive or reactive protocols. Reactive protocols executed all these steps on demand as when required that is why called on-demand protocols. At the mean time these protocols put together the operations and build up the route and then maintain it until the schedule is completed. Calculations are dynamic and onboard that is why reactive protocols are considered as the bottleneck for swift handoff. Proactive protocols execute all these steps before the handoff as the threshold frequency is tends to meet. As these protocols are relying on periodic broadcast of all existing options. Due to its broadcast nature proactive protocols are suffering from bandwidth overhead [3]. For better QoS reducing reactive and proactive protocols handover latency is compulsory. We are in the desire of an ideal scheme which can be a recipe in VHO and improving QoS by avoiding all the successors of latency like jitter, ping-pong effect, triangular routing and packet loss.

\section{Justification}

In our designed model the handoff phases will be managed initial three phases in proactive and later three phases in reactive mode as shown in the diagram. The mobile node will be either in dynamic or static position. But due to diverse form of networks availability the mobile node will be capable to use any network from $1 \mathrm{G}, 2 \mathrm{G}, 2.5 \mathrm{G}, 3 \mathrm{G}$ up to $4 \mathrm{G}$ after finding the appropriate RSSI value with affordable cost and satisfied services the mobile node will then in stage of vertical handoff initiation otherwise the node will keep its session on. When the mobile node initiates the handoff process it will first/proactively execute the three phases triggering, discovery and authentication, before leaving the current access point in which the mobile node is keep its session on. This phenomenon is called soft handoff. Until and unless the three defined phases are not executed the mobile node will then leave the current access point and start processing just the last three phases of handoff i.e. association, IP acquisition and house agent registration. This phenomenon is called hard handoff. In the last the new session is established with minimum time of handoff.

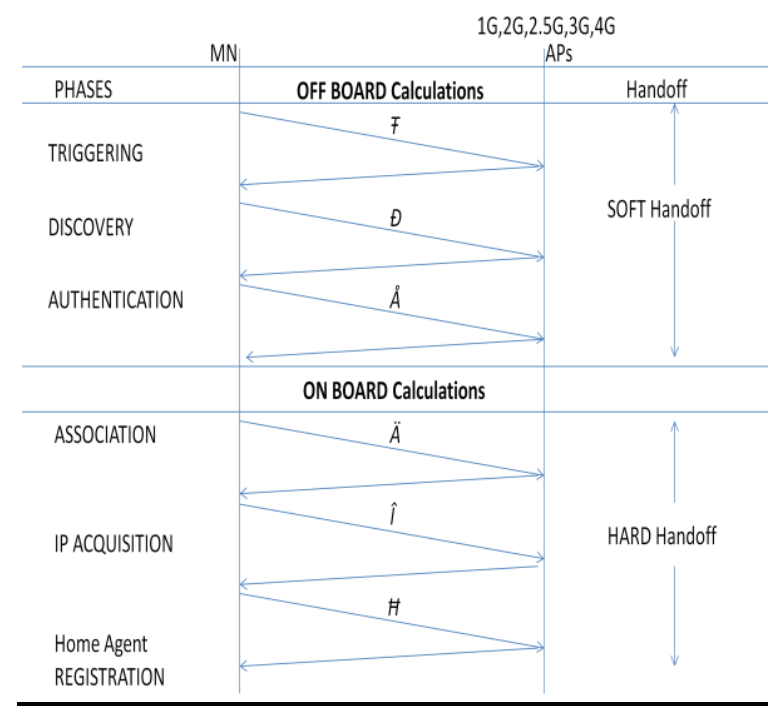

Figure1.3 Conceptual model

Here we have distributed the handoff phases in the following manner shown in the table.

\begin{tabular}{|l|c|c|}
\hline PHASES & PROACTIVE & REACTIVE \\
\hline Triggering & $\checkmark$ & $\mathbf{x}$ \\
\hline Discovery & $\checkmark$ & $\mathbf{x}$ \\
\hline Authentication & $\checkmark$ & $\mathbf{x}$ \\
\hline Association & $\mathbf{x}$ & $\checkmark$ \\
\hline $\begin{array}{l}\text { IP Acquisition } \\
\text { HA } \\
\text { Registrations }\end{array}$ & $\mathbf{x}$ & $\checkmark$ \\
\hline
\end{tabular}

Table 1.1 Parameters /Phases distribution table

\section{CONTROL FLOW DIAGRAM}

The mobile node while floating in the already availed network resources also seeking for the best quality of services networks with affordable cost with defined threshold of RSSI value[14]. This activity is called RSSI monitoring. If the seized RSSI value is less than the threshold value the mobile node then keep its old session on else control flow to trigger the option of the available access point. Once the option is triggered the mobile node then start discovery of the access point other features and process the authentication phase is processed by the node as well. Until this point of operation the control of the mobile node is divided with two access points one old other new one searched now.

Now the control concentrated wholly solely on the new access points while leaving the forerunner access point operating the rest three phases of handoff association, IP acquisition and House Agent Registration[14][15]. 


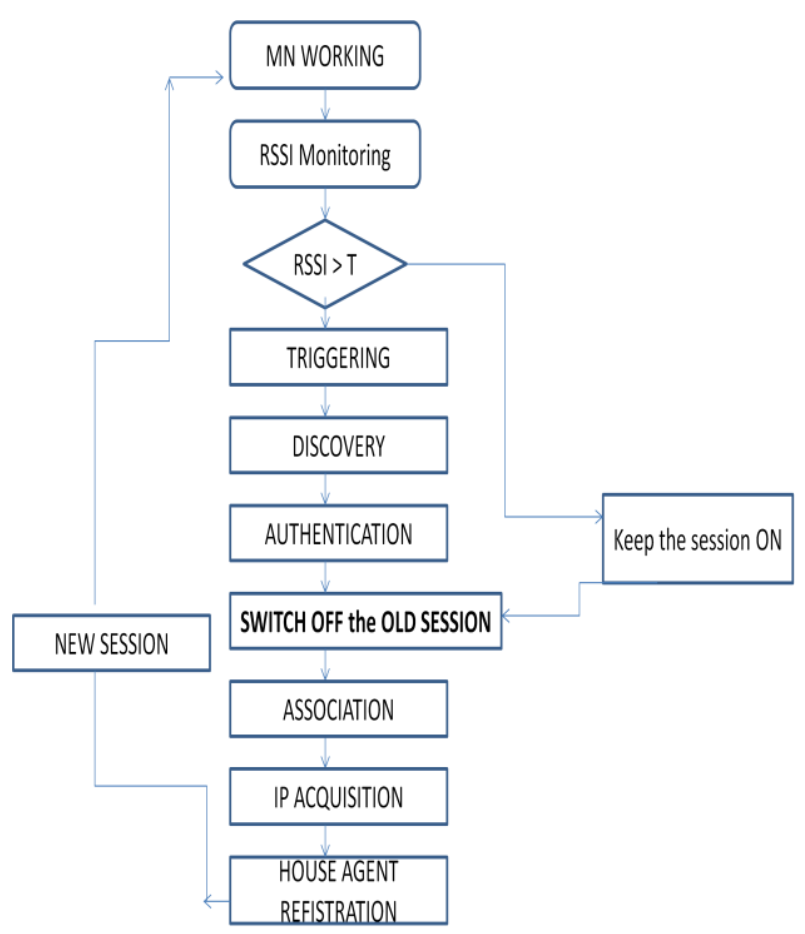

Figure 1.4 Control Flow Diagram

\section{MATHEMATICAL CALCULATION}

While switching from one network to another it is mandatory to execute the main six phases of handoff which are HA registration, IP acquisition, association, authentication, discovery and Triggering in any fashion according to the protocol used or implemented either reactive or proactive. In proactive protocol all the phases are executing before the handoff by the mobile node while switching from one network to another, while in reactive all the phases are executing after the demand or request is processed by the mobile node for handoff. In each and every step there must be time consumption according to the on ground situation of handoff. Whatever the sequence of steps may be used but the average calculated time will be the sum of the time consumed by each step.

Either REACTIVE or PROACTIVE Protocol time consumption

$\Delta V H O$ time $(V t)=(\Delta$ F) time $+(\Delta \boxminus)$ time $+(\Delta A)$ time $+(\Delta \ddot{A})$ time $+(\Delta \hat{I})$ time $+(\Delta H)$ time

(1)

In our proposed model we will divide the six main handoff phases into two categories for execution, they are reactive and proactive. Triggering, discovery and authentication will be executed in proactive manner while association, IP acquisition and house agent registration will be executed in reactive manner. So we will reduce the average time of handoff calculation in this manner as shown in the mathematical calculation.

\section{Proposed HYBRID Model time consumption}

Vertical Handoff initiation time $(\mathrm{Vt1})=$

$(\Delta$ F) time $+(\Delta Đ)$ time $+(\Delta \AA)$ time

(Proactive phases time)
Vertical Handoff execution time $(\mathrm{Vt} 2)=$

$(\Delta \ddot{A})$ time $+(\Delta \hat{I})$ time $+(\Delta H)$ time

(Reactive phases time)

Resultant Handoff time $(r V t)=V t-v t 1$.

(From equation 1 and 2)

ALGORITHM Preferences (Triggering 7 ), Discovery $Ð$, Authentication $\AA$, Association $\ddot{A}, I P$ acquisition $\hat{I}$, Home Agent Registration H, RSSI R)

1: Different Networks broadcast (NW1, NW2, NW3......NWn) their self for the mobile nodes (MN1, MN2, MN3 .....MNn)

$\mathrm{R}$ :> threshold $\{\mathrm{RSSI}$ value is suitable, no need for VHO $\}$

IF R $\rightarrow$ threshold $\{\mathrm{R} 1$ is RSSI value for VHO initiation $\}$

2: THEN

DO \{execute triggering, discovery and Authentication

3: Step1 7 AND activating the handoff steps process as the $\mathrm{R}$ tends to threshold

4: Step2 $Ð$ AND \{searching the appropriate RSSI value

5: Step $3 \AA \quad$ authenticate the mobile node

6: ELSE proceed within the same network

7: End if

8: end do

9: EXIT

10: IF STEPS 1, 2, 3 executed

11: THEN do

12: Step4 $\AA$ AND \{associate the network with new mobile node\}

13: Step5 $\hat{I}$ AND \{acquiring IP from care of address list

14: Step6 $H$ \{registration with home agent $\}$

END DO, RETURN R, EXIT.

\section{VALIDATION AND EVALUATION}

The grounding attention of this work is to build a hybrid model for vertical handoff to reduce the latency while evaluating reactive AODV, proactive OLSR and Hybrid TORA. We utilized OPNET simulator $\mathbf{1 4 . 5}$ versions to produce our results. 


\section{SIMULATION Environment}

Here in our ongoing scenario, the mobile node with multiple interfaces will have the capability to go for the most suitable access point (to avail the best quality of services within affordable cost) surrounded by all the available networks. The available networks will be from any generation network like $1 \mathrm{G}, 2 \mathrm{G}, 2.5 \mathrm{G}, 3 \mathrm{G}$ and $4 \mathrm{G}$.

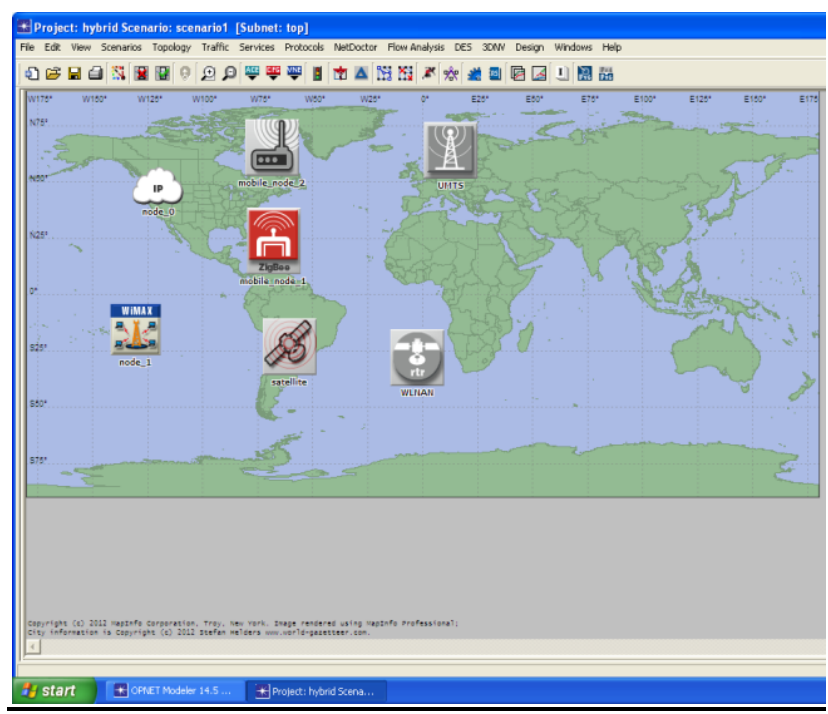

Figure 1.5 Simulation environment

\section{Latency calculation parameters and results}

In our simulation, the latency assessment is carried out by means of the following parameters. They are throughput, packet delivery ratio and end-to-end delay.

\section{Throughput}

Throughput is packets received by the destination from source totally and successfully. After analyzing the simulations results we found that hybrid protocol TORA showing the highest throughput which validates the effectiveness of the protocol. In contrast both reactive AODV and proactive OLSR lying in the bottom of the graph their results overlapping each other .

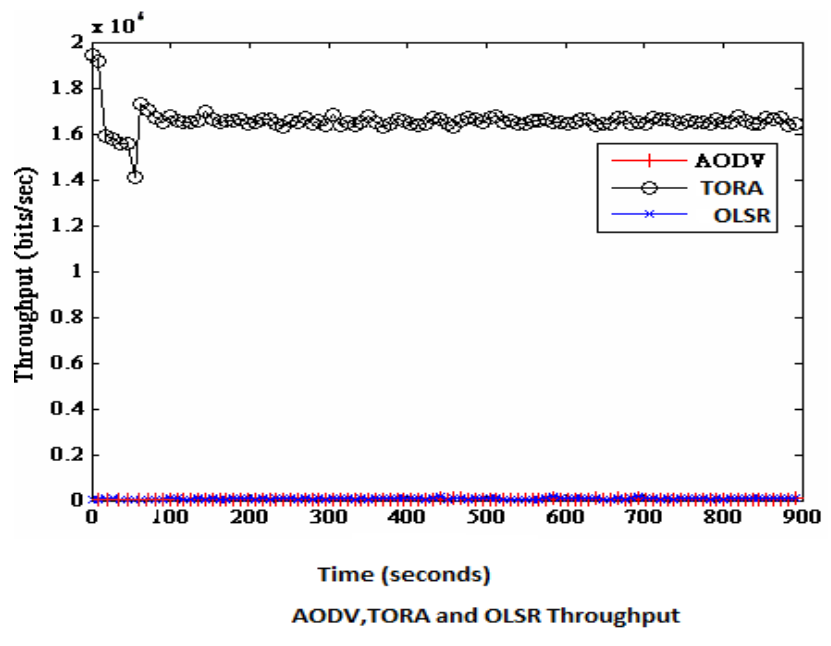

\section{Packet delivery ratio}

Packet delivery ratio is the proportional ratio of packets generated and sends by the source at the same time the ratio of packets to be received by the destination (sink).Again here the hybrid protocol TORA showing the highest packet delivery ratio in comparison with reactive AODV and proactive OLSR.

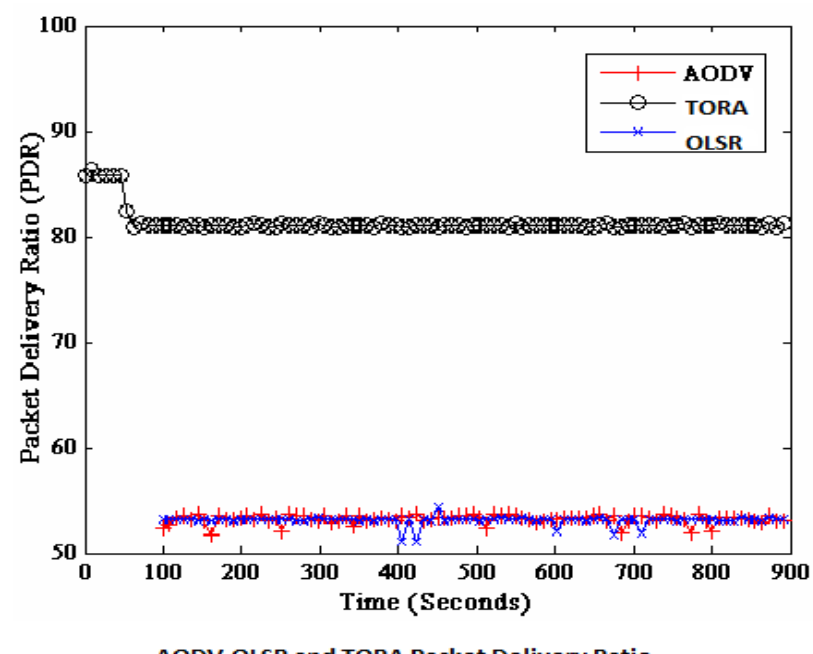

AODV,OLSR and TORA Packet Delivery Ratio

\section{CONCLUSION AND FUTURE WORKS}

After successful identification of latency causes and proposing the remedy in the form of hybrid model the authors will work for the authentication and packet loss issues which can be tackled now easily as the latency issue is addressed and resolved.

\section{REFERENCES}

[1] Mohammad Faisal and Muhammad nawaz khan "An enhanced Scheme for Reducing Vertical handover latency “,IJACSA, Vol. 3, No. 1, February 2012.

[2] Muhammad Nawaz Khan, Ishtiaq Wahid, Gulzar Mehmood "A Global Mobility Management Scheme for Reducing Overall Handover Latency in IP based VANETs", IJASUC, Vol.3, No.1,February 2012.

[3] Salima Hamma, Eddy Cizeron, Hafiz Issaka \& Jean-Pierre Guedon" Performance evaluation of reactive and proactive routing protocol in IEEE 802.11 ad hoc network", ITCom 06 -next generation and sensor networks, Boston : United States (2006)" DOI : 10.1117/12.686251.

[4] Gabriele Tamea, Anna Maria Vegni, Tiziano Inzerilli, Roberto Cusani " A Probability based Vertical Handover Approach to Prevent Ping-Pong Effect" 978-1-42443584- 5/09/\$25.00 @ 2009 IEEE, ISWCS 2009.

[5] Hoyeon Lee, Bongkyo Moon, and A. H. Aghvami "Enhanced SIP for Reducing IMS Delay under WiFi-toUMTS Handover Scenario The Second International Conference on Next Generation Mobile Applications, Services, and Technologies "978-0-7695-3333-9 108 $\$ 25.00$ (c) 2008 IEEE .

[6] Jaeho Jo and Jinsung Cho "A Cross-layer Vertical Handover between Mobile WiMAX and 3G Networks"978-1-4244-2202- 9/08/\$25.00 @ 2008 IEEE. 
[7] Neila KRICHENE and Noureddine BOUDRIGA "Securing roaming and vertical handover in fourth generation networks "Third International Conference on Network and System Security" 978-0-7695-3838-9/09 $\$ 26.00$ (C) 2009 IEEE .

[8] Pedro J. Fern'andez Ruiz Cristian A. Nieto Guerra Antonio F. G'omez Skarmeta "Deployment of a Secure Wireless Infrastructure oriented to Vehicular Networks", 2010, 24th IEEE International Conference on Advanced Information Networking and Applications",

[9] Chan-Kyu Han, Hyoung-Kee Choi,Jung Woo Baek, Ho Woo Lee "Evaluation of Authentication Signaling Loads in 3GPP LTE/SAE Networks", 2009 IEEE 34th Conference on Local Computer Networks (LCN 2009), Zürich, Switzerland; 20-23 October 2009 978-1-42444487-8/09/\$25.00 @2009 IEEE.

[10] Shih-Jung Wu "A New Integrated Mobile Architecture for Heterogeneous Wireless Networks", 2010 Sixth International Conference on Intelligent Information Hiding and Multimedia Signal Processing” 2010 IEEE.

[11] Wafaa Bou Diab and Samir TohmeSeamless "Handover and Security Solution for Real-Time Services" 2009, 11th IEEE International Symposium on Multimedia 9780-7695-3890-7/09 \$26.00 @ 2009 IEEE.
[12] Ali Al Shidhani and Victor C. M. Leung "Reducing Reauthentication Delays during UMTS-WLAN Vertical Handovers" 978-1-4244-2644-7/08/\$25.00 (2008 IEEE.

[13] Kun Zhu, Dusit Niyato, Ping Wang, Ekram Hossain, andDong In Kim "Mobility and Handoff Management in Vehicular Networks" Wireless Communication Mobile Computing 2009, online in Wiley Inter Science, DOI: 10.1002/wcm.0000.

[14] Sabina Baraković, Suad Kasapović, and Jasmina Baraković " Comparison of MANET Routing Protocols in Different Traffic and Mobility Models" Telfor Journal, Vol. 2, No. 1, 2010.

[15] Sourav Dhar, Amitava Ray, Rabindranath Bera " design and simulation of vertical handover algorithm for vehicular communication" International Journal of Engineering Science and Technology. Vol. 2(10), 2010, 5509-5525.

1Heterogenous Networks

2OSI (Open system interconnection)

3 IMS (IP Multimedia system)

4 SIP (Session Initiation Protocol) 